\title{
Tannins and Related Compounds in Callus and Root Cultures of Cornus capitata
}

\author{
Norie TANAKA*, Koichiro SHIMOMURA**, Takashi KAMIYA***, \\ Toshiaki KAYANO ${ }^{+}$and Kanji ISHIMARU* \\ *Department of Applied Biological Sciences, Faculty of Agriculture, Saga University, \\ 1 Honjo, Saga 840, Japan \\ **Tsukuba Medicinal Plant Research Station, National Institute of Health Sciences, \\ 1 Hachimandai, Tsukuba, Ibaraki 305, Japan \\ ${ }^{* * *}$ Chichibu Onoda Cement Corporation, Tsukuba Biotechnology R\&D Center, 25-13, \\ 1-Chome Kannondai, Tsukuba, Ibaraki 305, Japan \\ ${ }^{\dagger}$ Department of Cell Biology, National Institute of Agrobiological Resources, 1-2, \\ 2-Chome Kannondai, Tsukuba, Ibaraki 305, Japan
}

Received 17 September 1996; accepted 9 January 1997

\begin{abstract}
Callus and adventitious root cultures of Cornus capitata were established and the production of tannins and related compounds was determined in various culture conditions (media, plant growth regulators, light, etc.). Calli produced mainly procyanidins such as (+)-catechin and procyanidin B-3 on Murashige and Skoog (MS) and Woody Plant (WP) media. The maximum content of $(+)$-catechin $(0.1 \%$, as dry weight) was observed in the calli cultured on WP medium supplemented with $0.1 \mathrm{mg} / \mathrm{l} 2,4$-dichlorophenoxyacetic acid (2, 4-D) and $0.1 \mathrm{mg} / l$ benzyladenine (BA), or $2.0 \mathrm{mg} / l$ naphthaleneacetic acid (NAA) and $0.1 \mathrm{mg} / l \mathrm{BA}$ in the light. In contrast, adventitious roots cultured in MS liquid medium supplemented with $3.0 \mathrm{mg} / l$ indoleacetic acid (IAA) produced a high level of gallotannins such as 1,2,3,6-tetra- $O$-galloyl- $\beta$-D-glucose and $1,2,3,4,6$ penta- $O$-galloyl- $\beta$-D-glucose.
\end{abstract}

\section{Introduction}

Cornaceous plants are well known to contain large amounts of polyphenols, e.g. tannins (both hydrolysable and condensed types). In some medicinal and horticultural plants of this genera such as Cornus officinalis Sieb. et Zucc. [1] and C. kousa Buerge ex Hance [2], biotechnological approaches (callus and cell cultures) have been successful in the production and biosynthetic regulation of tannins. By HPLC analysis of phenolics in leaves of eight cornaceous plants, i.e. C. officinalis, C. kousa var. chinensis, C. kousa var. 'Milky Way', C. kousa var. Gold Star', C. kousa var. 'Satomi', C. kousa var. 'Snowboy', C. capitata 'Mountain Moon' and C. drummodii 'Eddie's White Wonder', C. capitata was clarified to be richest in tannin constituents [3]. C. capitata decorates the mountainsides in the Himalayas with its bright yellow four bracts, and is popular as a horticultural plant. However, the detailed biochemical studies of its constituents and tissue culture experiments have not been reported. In the present study, we established both callus and adventitious root cultures of this plant and examined their phenoic secondary metabolites produced under various conditions.

\section{Materials and Methods}

\section{1 Plant material and induction of the callus}

Leaf segments of Cornus capitata 'Mountain Moon' subcultured on Woody Plant (WP) [4] medium were placed on Murashige and Skoog (MS) [5] solid medium (solidified with $2.5 \mathrm{~g} / l$ gelrite) supplemented with various combinations of 2,4-dichlorophenoxyacetic acid (2,4-D), naphthaleneacetic acid (NAA), indoleacetic acid (IAA) and benzyladenine (BA) (Table 1). After 2 months of culture in the dark, the calli derived from the segments on four media (A, B, D and $\mathrm{H})$ were individually transferred to MS and WP solid media containing the same four combinations of growth regulators. They were subcultured at 8 week intervals in the light or dark over a half year.

MS and WP solid media used for the experiment contained $30 \mathrm{~g} / l$ and $20 \mathrm{~g} / l$ sucrose, respectively. All media were adjusted to $\mathrm{pH} 5.7$ before autoclaving at $121{ }^{\circ} \mathrm{C}$ for $15 \mathrm{~min}$. Cultures were placed in the light (3000 lux, $16 \mathrm{hr}$ light per day) or dark at $25^{\circ} \mathrm{C}$. Pieces of calli were cultured in sterile petri dishes $(9 \mathrm{~cm}$ in the diameter, three pieces of the calli per one petri 
dish) containing $25 \mathrm{~m} l$ medium. Data shown in Figs are the mean of 3 samples.

\section{2 Growth and tannin production of the callus}

Calli were harvested and the growth [dry weight $(\mathrm{dw})]$ and the production of nine phenolics \{gallic acid (1), $\beta$-glucogallin (2) [6], 1,2,6-tri- $O$-galloyl- $\beta$-D glucose (3), 1, 3, 6-tri- $O$-galloyl- $\beta$-D-glucose (4), 1, 2 , 3 , 6-tetra- $O$-galloyl- $\beta$-D-glucose (5), 1, 2, 3, 4, 6-penta - $O$-galloyl- $\beta$-D -glucose (6) $[7,8],(+)$-catechin (7), $(+)$-gallocatechin $(8)$ and procyanidin B-3 $[(+)$-cate-

\section{Table 1.}

Combination of plant growth regulators in MS solid medium used for the induction of Cornus capitata callus.

\begin{tabular}{ccccc}
\hline & $\begin{array}{c}2,4-\mathrm{D} \\
(\mathrm{mg} / l)\end{array}$ & $\begin{array}{c}\mathrm{NAA} \\
(\mathrm{mg} / \mathrm{l})\end{array}$ & $\begin{array}{c}\mathrm{IAA} \\
(\mathrm{mg} / l)\end{array}$ & $\begin{array}{c}\mathrm{BA} \\
(\mathrm{mg} / l)\end{array}$ \\
\hline $\mathrm{A}$ & 0.1 & & & \\
$\mathrm{~B}$ & 0.1 & & & 0.1 \\
$\mathrm{C}$ & 1.0 & & & \\
$\mathrm{D}$ & 1.0 & & & 0.1 \\
$\mathrm{E}$ & & 0.5 & & \\
$\mathrm{~F}$ & & 0.5 & & 0.1 \\
$\mathrm{G}$ & & 2.0 & & \\
$\mathrm{H}$ & & 2.0 & & 0.1 \\
$\mathrm{I}$ & & & 3.0 & \\
$\mathrm{~J}$ & & & 3.0 & 0.1 \\
\hline
\end{tabular}

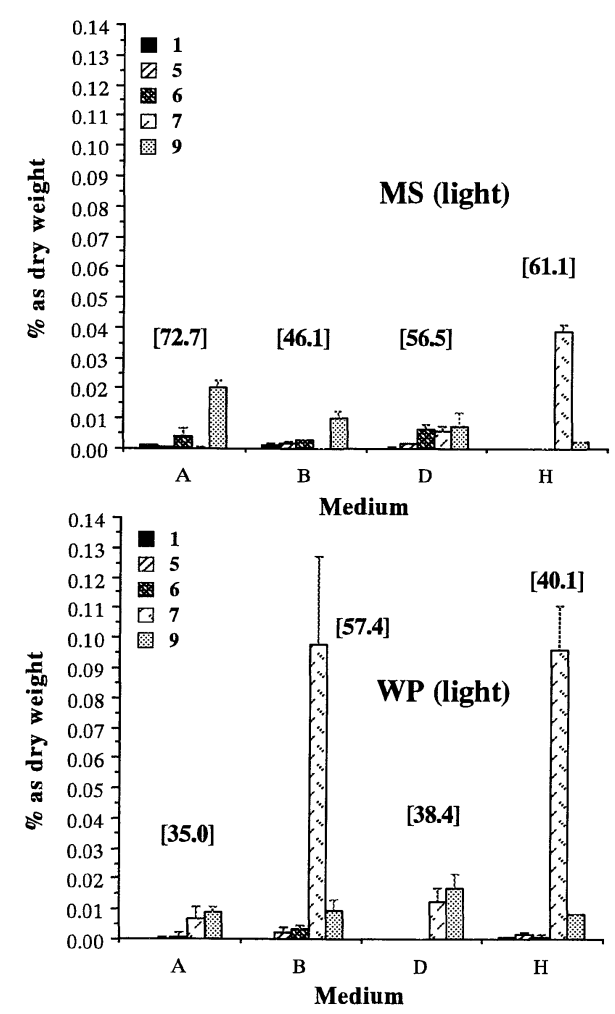

chin- $(4 \alpha \rightarrow 8)-(+)-$ catechin] (9) [9]\} were determined by HPLC.

\section{3 Establishment of adventitious root cultures and the tannin production}

Roots of the in vitro plantlets cultured on WP medium for 8 weeks were cut off, inoculated into MS and WP liquid medium $(50 \mathrm{~m} l$ in $100 \mathrm{~m} l$ Erlenmeyer flask) supplemented with $3.0 \mathrm{mg} / l$ IAA and subcultured on a rotary shaker $(100 \mathrm{rpm})$ at 8 week intervals in the dark over a half year. The root cultures were used for the determination of the growth and contents of phenolic compounds (1-9) (culture period, 14 weeks; harvested at every 2 weeks).

\section{4 Quantitative determination of tannins by HPLC}

Lyophilized samples (calli and roots, ca. 20-30 mg) were mashed and extracted with $\mathrm{MeOH}(2 \mathrm{~m} l)$ for 16 hr at room temperature. Each extract, after filtration through a Millipore filter $(0.45 \mu \mathrm{m})$, was subjected to HPLC analysis: column, TSK gel ODS 80Ts (4.6 $\mathrm{mm} \times 250 \mathrm{~mm})$; mobile phase, $1 \mathrm{mM}$ tetrabutylammonium chloride (adjusted to $\mathrm{pH} 2.9$ with $\mathrm{AcOH}$ ) $-\mathrm{CH}_{3}$ $\mathrm{CN}(9: 1 \rightarrow 1: 4$, in $30 \mathrm{~min}$.); flow rate, $0.6 \mathrm{ml} / \mathrm{min}$.; column temperature, $40{ }^{\circ} \mathrm{C}$; detection, $280 \mathrm{~nm}$ (UV); $\mathrm{R}_{\mathrm{t}}$ (min.), 2 (6.3), 1 (8.5), 8 (11.2), 9 (16.1), 7 (17.1), 3 (19. 2), 4 (20.5), 5 (22.8) and 6 (23.8).

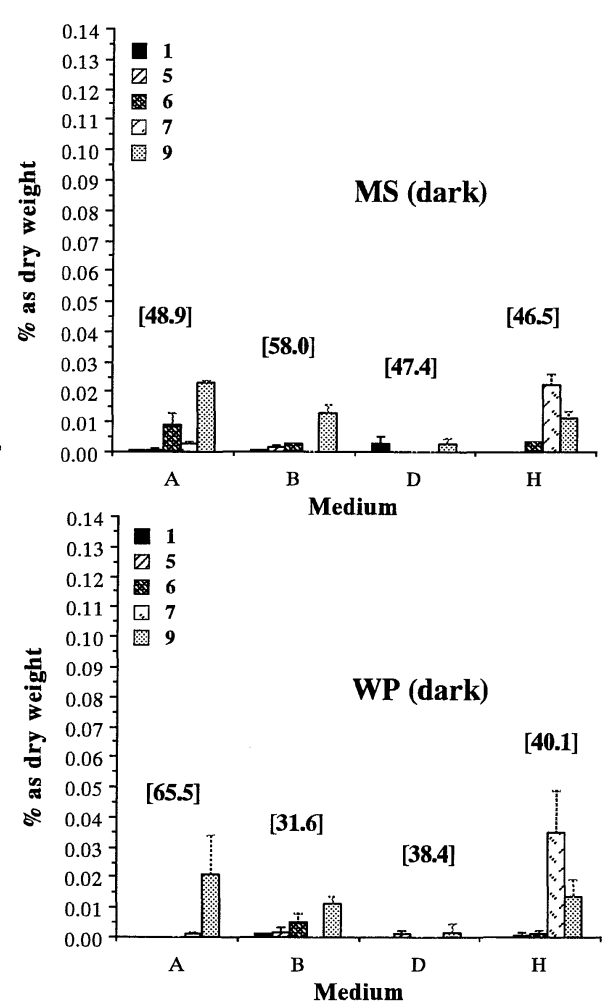

Fig. 1 Growth and polyphenol contents of Cornus capitata calli cultured on MS and WP solid media.

[ ]: dry weight of callus (mg). Growth regulators contained in media: $\mathrm{A}, 0.1 \mathrm{mg} / \mathrm{l} 2$, $4-\mathrm{D}, \mathrm{B}, 0.1 \mathrm{mg} / \mathrm{l} 2,4-\mathrm{D}$ and $0.1 \mathrm{mg} / l \mathrm{BA} ; \mathrm{D}, 1.0 \mathrm{mg} / l 2,4-\mathrm{D}$ and $0.1 \mathrm{mg} / l \mathrm{BA} ; \mathrm{H}, 2.0 \mathrm{mg} /$ $l \mathrm{NAA}$ and $0.1 \mathrm{mg} / l \mathrm{BA}$

(Bars represent standard errors.) 


\section{Results and Discussion}

C. capitata leaf explants for callus induction were prepared from in vitro plantlet subcultured on WP solid medium (the initial medium for establishment of the shoot cultures was BW medium [10]) [3]. From all leaf segments cultured on MS solid medium supplemented with various combinations of $2,4-D, N A$, IAA and BA, calli were derived (Table 1). On the media with 2, 4-D and NAA (media A-H) only callus formation was promoted, but on media with IAA (media I and J) adventitious roots were also derived from the surface of calli. Among ten media tested, good callus formation was observed on media with 2 , 4-D, particularly when combined with BA (media B and $\mathrm{D})$.

For the determination of growth and tannin production of C. capitata calli, two basal solid media (MS and WP) were used under the light or dark with four combinations of growth regulators $(A, B, D$ and $H)$, making 16 types of culture conditions. The results are shown in Fig. 1. There was little difference in the growth $(\mathrm{dw})$ of the calli cultured in these conditions. Among 9 phenolics (1-9), only five compounds (1, 5-7 and 9) were detected in the callus cultures (Fig.1). The contents of procyanidins [monomeric (7) and

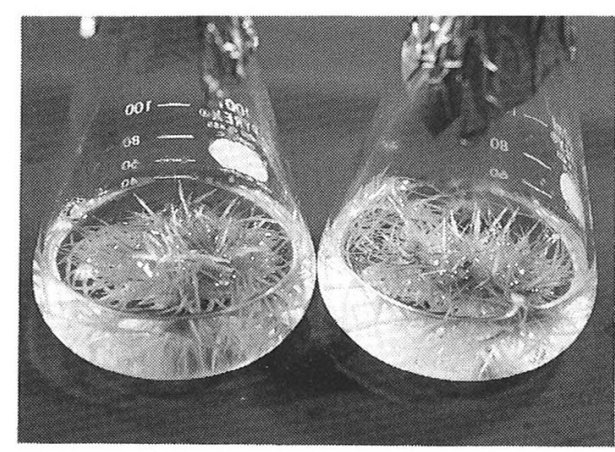

Fig. 2 Adventitious root cultures of Cornus capitata cultured in MS (left) and WP (right) liquid media with $3.0 \mathrm{mg} / l \mathrm{IAA}$ in the dark.

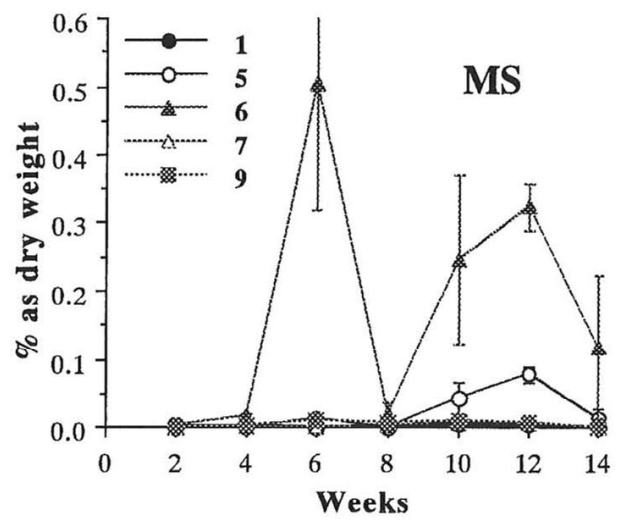

dimeric (9) compounds] were higher than those of galloylglucoses $(\mathbf{1}, \mathbf{5}$ and $\mathbf{6})$. The maximum content of 7 (ca. $0.10 \%$ as dw) was observed on two types of WP media (B and $\mathrm{H}$ combinations, see Table 1) under light. Compared to tannin contents in leaves of the parent plant $(1,0.01 \%$; 5 , trace; $6,1.46 \% ; 7,0.36 \% ; 8,0.06 \%$ $; 9,0.05 \%$, as dw) [3], those observed in callus cultures were fairly low.

Adventitious root cultures of $C$. capitata were also established (Fig. 2; see Materials and Methods) and the polyphenol production in the tissues was determined. The roots grew well showing the maximum level (dw/ a flask) at week $8(415 \mathrm{mg})$ in MS medium and week $14(382 \mathrm{mg}$ ) in WP medium, respectively (Fig. 3). The reason for the slight decrease in the dry amount of the roots cultured in MS medium at week 6 is not clear. Possibly, the roots which have not grown well were harvested at week 6 by chance. However, this decrease was found to coincide with the increase of galloylglucose contents (see below).

Although the root cultures in MS and WP media showed similar growth patterns (Fig. 3), tannin contents were greatly affected by media constituents (Fig. 4). In the roots cultured in MS medium with 3.0

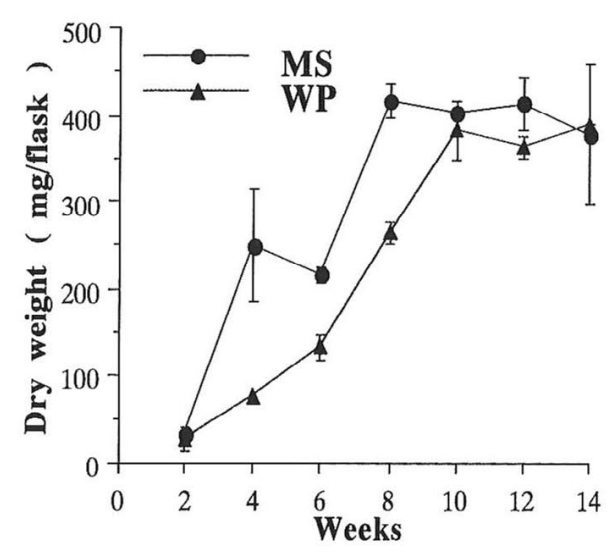

Fig. 3 Growth of the adventitious roots of Cornus capitata cultured in MS and WP liquid media with $3.0 \mathrm{mg} / l$ IAA in the dark.

(Bars represent standard errors.)

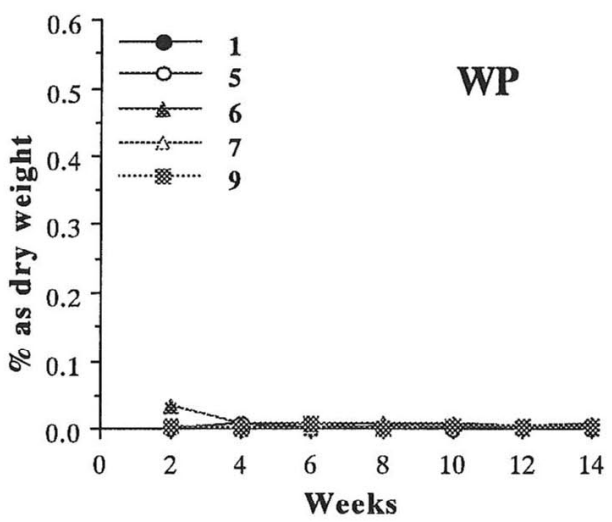

Fig. 4 Contents of phenolic compounds in adventitious roots of Cornus capitata cultured in MS and WP liquid media with $3.0 \mathrm{mg} / \mathrm{l}$ IAA in the dark.

(Bars represent standard errors.) 
$\mathrm{mg} / \mathrm{l}$ IAA, high galloylglucoses (5 and 6) levels were observed during the culture period with a plateau growth i.e. weeks 4 to 6 and 8 to 14 . The maximum content of $6(0.5 \%$ as $\mathrm{dw})$ was observed at week 6 . In contrast, during the culture period displaying a rapid growth (weeks 2 to 4 and 6 to 8), the tannin contents in the roots were low, or declined. When cultured in WP medium, the adventitious roots produced a poor level of tannins during the whole culture period.

The following results are biosynthetically noteworthy; 1) C. capitata callus produced mainly procyanidins (7 and 9 ) regardless of culture conditions such as medium (MS or WP) and illumination; 2) $C$. capitata adventitious roots produced mainly hydrolysable tannins (galloylglucoses, $\mathbf{5}$ and $\mathbf{6}$ ), and the productivity was affected by medium constituents. These results indicate the promotion of the activity of the enzymes for galloylglucose biosynthesis in organ tissues of C. capitata.

In this experiment, we succeeded, for the first time, in tannin production in adventitious roots of woody plants in liquid culture. Both callus and root cultures of $C$. capitata will offer good systems for biochemical studies of hydrolysable and condensed tannins.

\section{Acknowledgements}

This work was supported in part by the subsidy from The San-Ei-Gen Foundation for Food Chemical Research and by a special coordination fund for promoting science and technology in the basic research core system by the Science and Technology Agency.

\section{References}

[1] Yazaki, K., Okuda, T., 1989. Plant Cell Reports, 8: 346-349.

[2] Ishimaru, K., Arakawa, H., Neera, S., 1993. Phytochemistry, 32: 1193-1197.

[3 ] Ishimaru, K., Tanaka, N., Kamiya, T., Sato, T., Shimomura, K., In Biotechnology in Agriculture and Forestry, 'Medicinal and Aromatic Plants $\mathrm{X}$, (in press).

[4] Lloyd, G.B., McCown, B.H., 1980. Int. Plant. Prop. Soc., 30: 421-427.

[5] Murashige, T., Skoog, F., 1962. Physiol. Plant., 15: 473-497.

[6] Kashiwada, Y., Nonaka, G., Nishioka, I., 1984. Chem. Pharm. Bull., 32: 3461-3470.

[7] Haddock, E.A., Gupta, R.K., Al-Shafi, S.M.K., Haslam, E., Magnolato, D., 1982. J. Chem. Soc. Perkin Trans. I: 2515-2524.

[ 8 ] Haddock, E.A., Gupta, R.K., Haslam, E., 1982. J. Chem. Soc. Perkin Trans. I: 2535-2545.

[9] Thompson, R. S., Jacques, D., Haslam, E., Tanner, R. J. N., 1972. J. Chem. Soc. Perkin Trans. I: 1387-1399.

[10] Sato, T., 1991. Bull. of For. and For. Prod. Res., 360: 35-119. 